\title{
A Theoretical Antioxidant Mechanism for Cytoprotective Effect of $p$-Acetamide- Salicylate Derivatives against Free Radical Initiator AAPH in Human Erythrocytes
}

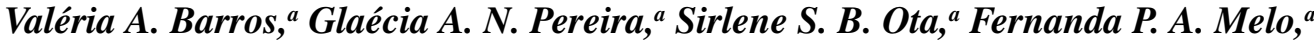 \\ Ana Carolina S. P. S. de Jesus, ${ }^{a}$ Anderson B. Lima, ${ }^{b}$ Albérico B. F. da Silva ${ }^{c}$ and \\ Rosivaldo S. Borges ${ }^{\circledR} *, a$
}

${ }^{a}$ Núcleo de Estudos e Seleção de Moléculas Bioativas, Instituto de Ciências da Saúde, Universidade Federal do Pará, CP 11101, 66075-110 Belém-PA, Brazil

${ }^{b}$ Centro de Ciências Biológicas e da Saúde, Universidade do Estado do Pará, 68458-100 Tucuruí-PA, Brazil

'Instituto de Química de São Carlos, Universidade de São Paulo, CP 780, 13560-970 São Carlos-SP, Brazil

\begin{abstract}
The molecular mechanism of cytoprotective effect on human erythrocytes of aminophenol and salicylates associated derivatives was related to their antioxidant capacity. The oxidative hemolysis induced by water-soluble free-radical initiator 2,2' -azobis-(2-amidine-propane)-dihydrochloride (AAPH) was inhibited by drug candidates named benzaminophen (BZL), salicytamide or 5-acetamide-salicylic acid (ASL), and salibenzamide or 5-benzamide-salicylic acid (BSL) when compared to their parents salicylic acid (SAC) and acetaminophen (ACP). Trolox (TLX) was the most powerful compound and used as positive control. BZL showed a potent effect followed by ACP $>$ BSL > ASL. SAC did not show protective effect in any evaluated concentrations. These results are in accordance with the molecular mechanism by using theoretical calculation of single electron transfers (SET), hydrogen atom transfers (HAT), and sequential proton loss electron transfer (SPLET) by means of DFT/B3LYP/6-31++G(d,p) level of theory. [1,5] Hydrogen shift between carboxyl and phenol moieties and electronic properties related to $\mathrm{p} K_{\mathrm{a}}$ and other physicalchemical properties can be involved. The molecular association approach provides protective compounds more effective than SAC.
\end{abstract}

Keywords: free-radical, erythrocytes, hemolysis, DFT, antioxidant, phenol derivatives

\section{Introduction}

Recent studies ${ }^{1}$ suggest that aggregation of platelets from patients with coronary artery and cerebrum-vascular disease may be resistant to low-dose aspirin treatment, which may promote plaque-associated thrombus formation. Aspirin blocks the platelet aggregation primarily by inactivating cyclooxygenase (COX) and also thromboxane A2 (TxA2) formation. ${ }^{2} \mathrm{~A}$ series of bioactive prostaglandin-like compounds (isoprostanes) have been discovered that are produced from arachidonic acid through a non-enzymatic process of lipid peroxidation, catalyzed by oxygen free-radicals. ${ }^{3}$ The increase of isoprostanes level on plasma has been shown in pathophysiological conditions associated with

*e-mail: rosborg@ufpa.br oxidative stress including diabetes, cigarette smoking, advanced age, hypercholesterolemia and hyper homocysteinemia. ${ }^{4-6}$ Some conditions can be blocked by vitamin E supplementation, a classical antioxidant. ${ }^{4}$

Reactive oxidants are produced on phagocytic cells inducing tissue damage in chronic inflammatory disorders and phenolic drugs can act as potential scavenger of some reactive species. ${ }^{7-10}$ Nevertheless, the low antioxidant capacity or other biological mechanisms may limit the molecule candidate application as drug. For example, acetyl salicylic acid may cause gastric irritation and its use is forbidden where such irritation must necessarily be avoided. In fact, its gastric irritation and intestinal bleeding can be initiated through three main mechanisms, i.e., an enzymatic process through prostaglandin inhibition, a direct action due to its low $\mathrm{p} K_{\mathrm{a}}$ among other salicylates and also oxidative stress conditions. ${ }^{11}$ 
Red blood cell (RBC) membrane is rich in polyunsaturated fatty acids, which are very susceptible to free-radical mediated peroxidation. So, the rate of freeradical generation from 2,2'-azobis-(2-amidine-propane)dihydrochloride (AAPH) can be easily controlled and measured. The hemolysis induced by AAPH provides a good approach for studying the free-radical induced membrane damage. ${ }^{12,13}$

Therefore, in the present work, an induced oxidative hemolysis of human RBC was used for determination of the inhibitory effect of $p$-aminophenol and salicylates association derivatives. The theoretical mechanisms for the antioxidant and protective properties were studied using molecular modeling. Our subject is the salicylate derivatives evaluation with free radical scavenging properties as an important strategy for the development of therapeutic candidates in aspirin resistance.

\section{Experimental}

\section{Drug design}

The derivatives were obtained using a rational drug design by molecular association and functional bioisosteric group substitution as previously reported. ${ }^{14}$ The acetamide moiety is changed to benzamide group. The structural properties of benzamidephenol (BZL), salicytamide or 5-acetamide-salicylic acid (ASL), a bioisosteric derivative of gentisic acid (GAC), and 5-benzamide-salicylic acid (BSL), were compared to their parent acetaminophen (ACP) and salicylic acid (SAC). Trolox (TLX), a water-soluble analogous of vitamin $\mathrm{E}$ was used as positive control against AAPH. All interactions with antioxidant defense was also studied. Our subject is the development of associated derivatives more effective than aspirin in the free-radical-induced hemolysis (Figure 1).

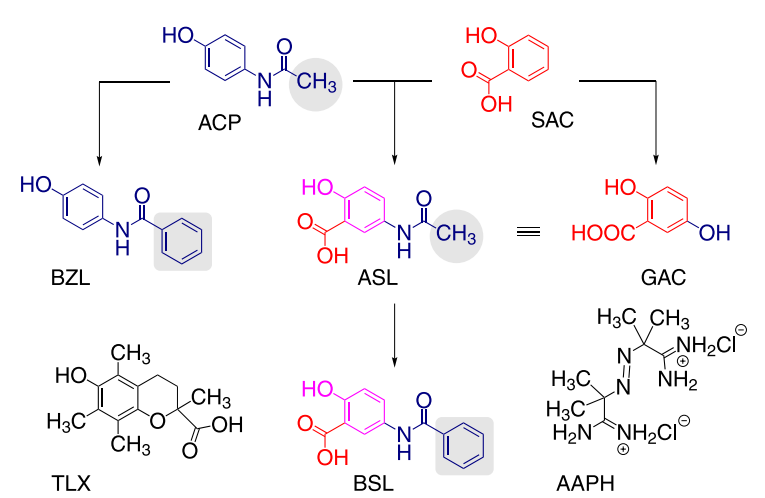

Figure 1. Structure of Trolox, AAPH and acetaminophen-salicylic acid derivatives.

\section{Materials and instruments}

Trolox, acetaminophen, salicylic acid and 2,2-azobis(2-amindino-propane)-dihydrochloride were purchased from Sigma-Aldrich company, St. Louis, MO, United States. Benzamidephenol, 5-acetamide-salicylic acid and 5-benzamide-salicylic acid were synthesized by acetylation and benzylation from $p$-aminophenol and 5 -amine-salicylic acid, as previously reported. ${ }^{14}$ Ultravioletvisible-near infrared (UV-Vis-NIR) spectrophotometer model Cary 6000i from Varian was used to determine the hemolysis level. Specifications: range $175-1800 \mathrm{~nm}$, resolution $<0.05 \mathrm{~nm}$ in UV-Vis, $<0.1 \mathrm{~nm}$ in NIR. It was also used a laboratory centrifuge Sigma 6-15, air-cooled, for universal high-volume use, with speed range 100 to $13500 \mathrm{rpm}$, accuracy $1 \mathrm{rpm}$.

\section{Erythrocytes}

Human blood was obtained from student volunteer donors at College of Pharmacy in Federal University of Pará. Blood was collected after obtaining informed consent, with local ethics committee approval under number 076/06 CEP-CCS/UFPA. Human red blood cells were separated from blood with ethylenediamine tetraacetic acid (EDTA) that was drawn from a healthy donor. The blood was centrifuged at $2000 \mathrm{rpm}$ for $10 \mathrm{~min}$ to separate the RBC from plasma, then the RBC was washed three times with phosphate-buffered saline (PBS) at $\mathrm{pH} 7.4$ which consists of $137 \mathrm{mM} \mathrm{L}^{-1} \mathrm{NaCl}, 2.7 \mathrm{mM} \mathrm{L}^{-1} \mathrm{KCl}, 8.1 \mathrm{mM} \mathrm{L}^{-1} \mathrm{Na}_{2} \mathrm{HPO}_{4}$ and $1.5 \mathrm{mM} \mathrm{L}^{-1} \mathrm{KH}_{2} \mathrm{PO}_{4}$ in distilled water. During the last washing, the cells were centrifuged at exactly $2000 \mathrm{rpm}$ for $10 \mathrm{~min}$ to obtain a constantly packed cell volume. ${ }^{15}$

\section{Determination of erythrocytes hemolysis}

The 5\% suspension of $\mathrm{RBC}$ in PBS (pH 7.4) was incubated under air atmosphere at $37^{\circ} \mathrm{C}$ for $5 \mathrm{~min}$, to which an aqueous solution of $70 \mathrm{mM}$ AAPH was added to initiate hemolysis. The reaction mixture was shaken gently while being incubated at $37^{\circ} \mathrm{C}$. The extent of the hemolysis was determined spectrophotometrically as described previously. ${ }^{16}$ Aliquots of the reaction mixture were taken out at appropriate time intervals, diluted with $0.15 \mathrm{~mol} \mathrm{~L}^{-1}$ of $\mathrm{NaCl}$ and centrifuged at $2000 \mathrm{rpm}$ for $10 \mathrm{~min}$ to separate the RBC. The absorbance A of the supernatant at $540 \mathrm{~nm}$ was measured. Similarly, the reaction mixture was treated with distilled water to make complete hemolysis and the absorbance B of the supernatant after centrifugation was measured at $540 \mathrm{~nm}$. The hemolysis percentage was calculated from the ratio of the measurements $(\mathrm{A} / \mathrm{B}) \mathrm{A} \times 100$. In the case of 
antioxidant experiments, the phenolic derivatives were added and incubated before addition of AAPH.${ }^{17}$ Every experiment was repeated three times and the results were reproducible within $p<5 \%$ detour.

\section{Molecular modeling}

All the calculations were performed with the Gaussian 03 molecular package. ${ }^{18}$ Prior to any density functional theory $(\text { DFT })^{19}$ calculations all structures were submitted to an initial parametric method $3(\mathrm{PM} 3)^{20}$ geometry conformational search. After that, all PM3 geometries were re-optimized with the Becke three-parameter Lee-Yang-Parr (B3LYP) hybrid functional ${ }^{21,22}$ by using a $6-31^{++} \mathrm{G}(\mathrm{d}, \mathrm{p})$ basis. ${ }^{23}$ The ionization potential (IP) was calculated as the energy difference between a neutral molecule and the respective cation free-radical (equation 1). The bond dissociation energies of the phenol group $\left(\mathrm{BDE}_{\mathrm{OH}}\right)$ or semiquinone formation was calculated as the energy difference between a neutral molecule and the respective semiquinone plus the hydrogen radical as defined in equation 2 .

$\mathrm{IP}=\mathrm{EArOH}^{++}-\mathrm{EArOH}$

$\mathrm{BDE}_{\mathrm{OH}}=\left(\mathrm{EArO}^{*}+\mathrm{EH}^{\circ}\right)-\mathrm{EArOH}$

IP and $\mathrm{BDE}_{\mathrm{OH}}$ were related to single electron transfers (SET) and hydrogen atom transfers (HAT) in equations 3 and 4, respectively; all molecules $(\mathrm{ArOH})$ are compared to Trolox (TLX).

$$
\begin{aligned}
& \mathrm{SET}=\left(\mathrm{ETLX}^{\cdot+}+\mathrm{EArOH}\right)-\left(\mathrm{ETLX}^{\left.+\mathrm{EArOH}^{+}\right)}\right. \\
& \mathrm{HAT}=\left(\mathrm{ETLX}^{*}+\mathrm{EArOH}\right)-\left(\mathrm{ETLX}^{\circ}+\mathrm{EArO}^{*}\right)
\end{aligned}
$$

The sequential proton loss electron transfer (SPLET) mechanism occurs when phenols lose a proton to a phenolate anion by an acid-base equilibrium (equation 5), following an electron transfer to form the phenoxyl radical and anion (equation 6).

$\mathrm{ArOH} \rightarrow \mathrm{ArO}^{-}+\mathrm{H}^{+}$

$\mathrm{SPLET}=\mathrm{EArO}^{*}-\mathrm{EArO}^{-}$

The relationship between SET, HAT, SPLET and hemolysis (\%) was used as an alternative to elucidate the antioxidant mechanism of these compounds.

\section{Results and Discussion}

\section{Determination of erythrocytes hemolysis}

The effects of phenolic drugs on AAPH-induced RBC hemolysis were searched by the hemoglobin absorbance and compared to TLX. Figure 2 shows that in the absence of AAPH, the RBC was stable and a little hemolysis took place in $2 \mathrm{~h}$. The concentration of TLX, ACP, and SAC were: $0.250,0.500$, and $1 \mathrm{mM} \mathrm{L}^{-1}\left(70 \mathrm{mM} \mathrm{L}^{-1} \mathrm{AAPH}\right)$, respectively. The addition of $70 \mathrm{mM}$ AAPH induced, after an inhibition period, a fast hemolysis. Other concentrations were utilized but not reported in this study. Also, the concentration of $70 \mathrm{mM}$ was reported previously. ${ }^{15}$

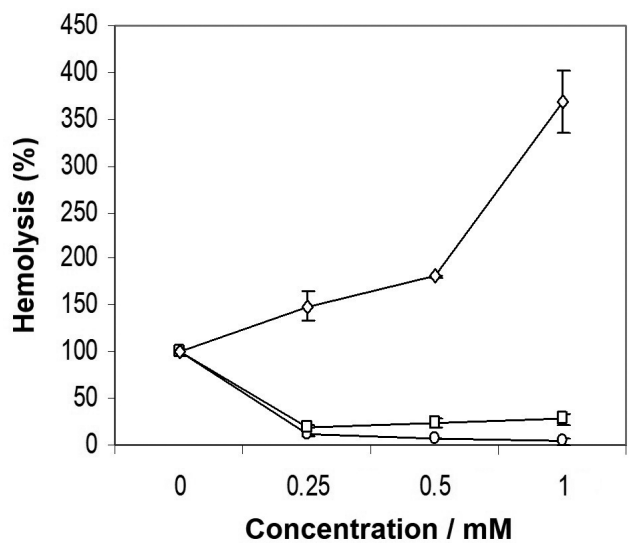

Figure 2. Results of AAPH-induced hemolysis of 5\% human RBC in $0.15 \mathrm{~mol} \mathrm{~L}^{-1} \mathrm{PBS}(\mathrm{pH} 7.4)$ under air atmosphere at $37^{\circ} \mathrm{C}$ by TLX $(O)$, $\operatorname{ACP}(\square)$, and $\operatorname{SAC}(\diamond)$. Data are expressed as the mean of three samples.

The pre-incubation with ACP or TLX inhibits AAPH-induced RBC hemolysis as a function of the drug concentration and SAC has no apparent effect. Compared with ACP, the classical antioxidant standard TLX exhibits a significant higher protection against AAPH-induced $\mathrm{RBC}$ hemolysis. The mean percentage of hemolysis for the $0.25,0.5$, and $1 \mathrm{mM}$ doses of the drugs showed that TLX decreases the hemolysis and present 7.40 of the amount of hemoglobin absorbance, while the same concentrations of ACP exerts an effect of 24.02. However, SAC does not protect the membrane against this degradative process in the range of the used concentrations (up to $1 \mathrm{mM}$ ) with mean of 232.95 .

From Figure 3, we can see that the rate of hemolysis and the inhibition percentage are correlated with the concentration of the associated derivatives between $p$-aminophenol and salicylates. The pre-incubation with ASL or BSL decreased AAPH-induced RBC hemolysis compared with $\mathrm{SAC}$ as a function of the drug concentration and BZL protects the membrane damage by AAPH-induced hemolysis in RBC. The concentration of BZL, ASL, and BSL were: $0.250,0.500$, and $1 \mathrm{mM} \mathrm{L}^{-1}\left(70 \mathrm{mM} \mathrm{L}^{-1}\right.$ AAPH). The mean percentage of hemolysis for the 0.25 , 0.5 , and $1 \mathrm{mM}$ doses of the drug candidates showed that BZL decreases the hemolysis level presenting 21.30 of 
the amount of hemoglobin absorbance, while the same concentrations of ACP exerts an effect of 24.02. ASL does not protect the membrane against this degradative process in the range of the used concentrations with 158.18. Nevertheless, BSL showed effective protection against membrane damage with 101.35 , compared with the prooxidant effect of SAC (232.95).

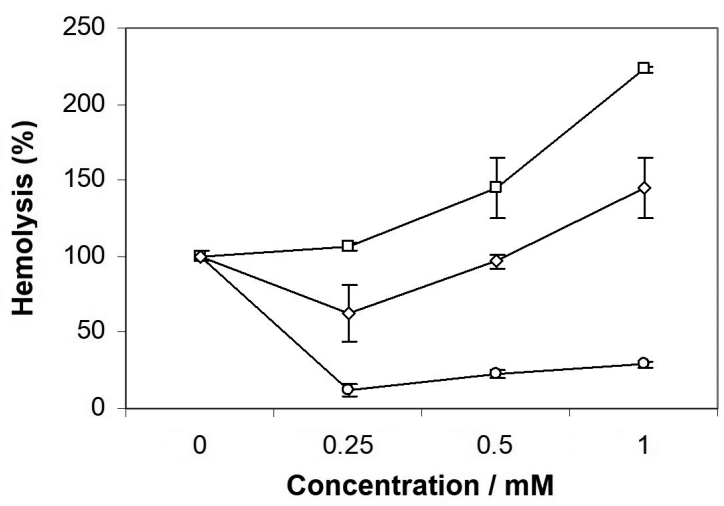

Figure 3. Results of AAPH-induced hemolysis of 5\% human RBC in $0.15 \mathrm{~mol} \mathrm{~L}^{-1} \mathrm{PBS}(\mathrm{pH} 7.4)$ under air atmosphere at $37^{\circ} \mathrm{C}$ by BZL $(\mathrm{O})$, $\operatorname{ASL}(\square)$, and BSL $(\diamond)$. Data are expressed as the mean of three samples.

The thermal decomposition of AAPH in the aqueous dispersion of $\mathrm{RBC}$ produces a radical $\left(\mathrm{R}^{*}\right)$. The reaction between $\mathrm{R}^{\cdot}$ and oxygen form an initiating radical (ROO') which can attack the polyunsaturated lipids (LH) in RBC membrane inducing lipid peroxidation. ${ }^{21}$ Since the lipid peroxidation is the main free-radical reaction and initiating radical could induce up to propagation reactions,${ }^{24}$ the RBC membrane is quickly damaged leading to hemolysis. Therefore, the hemolysis occurs by two mechanisms: in the first one, the $\mathrm{R}^{\cdot}$ and $\mathrm{ROO}^{*}$ radicals are formed via thermo-generation of free-radicals by AAPH. And in the second one, they are formed by endogenous antioxidants depletion in erythrocytes and they can trap the initiating and/or propagating radicals to inhibit the peroxidation of the polyunsaturated fatty-acid in the membrane of the erythrocytes. ${ }^{23}$
The structure-capacity relationship of $p$-aminophenol and salicylate association

In order to determine the effect of the structural characteristics of this kind of phenolic derivatives on their antioxidant or pro-oxidant capacity, theoretical calculations were carried out using the DFT/B3LYP method.

Phenolic compounds have been found to be effective antioxidants in biological systems and their effectiveness not only depends on the stability of phenoxyl radical formed in the reaction, but of their electron or hydrogen donating capacities. ${ }^{17,25,26} \mathrm{ACP}$ and SAC, although closely related to the molecular structure exert very different activities as free-radical scavengers. The ionization potential (IP) can indicate how ACP acts as a potential scavenger by single electron transfers (SET) due to the small hemolysis percentage, in contrast with SAC. In turn, ACP exhibits comparative scavenger capacity when compared to TLX.

Indeed, the antioxidant mechanisms of phenol derivatives include the hydrogen or electron transfers to a free-radical. The former is controlled by the bond dissociation energy and the latter is controlled by the IP or SET. So, the lower SET values are related to the higher electron-transfer ability. The electron transfer was calculated as the energy difference between the radical species and the neutral molecule.

The SET represents the easiness for an electron donation of the phenolic derivatives. Electron-donating groups at the ortho or para positions $\left(-\mathrm{CH}_{3},-\mathrm{O}^{-}\right.$, and $\left.-\mathrm{NHCOCH}_{3}\right)$ decrease the SET values resulting in a better scavenger capacity, while electron-withdrawing groups $(-\mathrm{COOH})$ increase the SET values resulting in a decrease of the scavenger capacity when compared to TLX. Nevertheless, in accordance with Table 1, ACP showed the lowest SET values in gas phase $\left(34.68 \mathrm{kcal} \mathrm{mol}^{-1}\right)$, while SAC showed the highest SET value $\left(57.31 \mathrm{kcal} \mathrm{mol}^{-1}\right)$ when compared to Trolox.

Table 1. Theoretical properties of electron and hydrogen specie transfers for phenolic derivatives

\begin{tabular}{|c|c|c|c|c|c|}
\hline Phenolic derivative & IP / ( $\left.\mathrm{kcal} \mathrm{mol}^{-1}\right)$ & $\mathrm{SET} /\left(\mathrm{kcal} \mathrm{mol}^{-1}\right)$ & $\mathrm{BDE} /\left(\mathrm{kcal} \mathrm{mol}^{-1}\right)$ & HAT / $\left(\mathrm{kcal} \mathrm{mol}^{-1}\right)$ & SPLET / $\left(\mathrm{kcal} \mathrm{mol}^{-1}\right)$ \\
\hline TLX & 136.71 & 0 & 60.36 & 0 & 38.43 \\
\hline $\mathrm{ACP}$ & 171.39 & 34.68 & 85.87 & 25.51 & 48.19 \\
\hline SAC & 194.03 & 57.31 & 104.82 & 44.46 & 76.54 \\
\hline BZL & 168.22 & 31.50 & 85.82 & 25.46 & 51.32 \\
\hline ASL & 175.10 & 38.39 & 100.07 & 39.71 & 70.50 \\
\hline BSL & 172.20 & 35.49 & 99.22 & 38.85 & 73.43 \\
\hline
\end{tabular}

IP: ionization potential; SET: single electron transfer; BDE: bond dissociation energy; HAT: hydrogen atom transfer; SPLET: sequential proton loss electron transfer; TLX: Trolox; ACP: acetaminophen; SAC: salicylic acid; BZL: benzaminophen; ASL: 5-acetamide-salicylic acid; BSL: 5-benzamide-salicylic acid. 
Furthermore, comparing the three phenolic drugs, SAC possessing a carbonyl ortho group to phenol is practically deprived of antioxidant capacity. ACP with an intermediary efficiency as antioxidant, has a substituent at the para position in relation to hydroxyl group. TLX, which is the most efficient of the three, possesses a methyl group, chromone ring at the ortho and para positions in relation to hydroxyl group. These SET values are in accordance with highest occupied molecular orbital (HOMO) energy, as previously reported by us. ${ }^{14}$

In accordance with the electron abstraction, the hydrogen abstraction showed that the $\mathrm{O}-\mathrm{H}$ moiety can be responsible for the antioxidant capacity by hydrogen abstraction due to its higher $\mathrm{HOMO}$ or lower SET and $\mathrm{BDE}_{\mathrm{OH}}$ or HAT values. Consequently, ACP and SAC have the HAT values related to TLX of 25.51 and $44.46 \mathrm{kcal} \mathrm{mol}^{-1}$, respectively.

In addition, in molecular association, the carbonyl electron-withdrawing group increased the IP, while the hydroxyl electron-donating group decreased the SET values, resulting in an increment of the scavenger capacity when compared to SAC. In Table 1, BZL showed a more scavenger capacity than ACP. The para-phenol acylamide group is common to the two compounds, but the phenyl group presented a smaller SET value $\left(31.50 \mathrm{kcal} \mathrm{mol}^{-1}\right)$ than the methyl group $\left(38.39 \mathrm{kcal} \mathrm{mol}^{-1}\right)$. Nevertheless, the molecular association of the $p$-aminophenol and salicylate structure under study, 5-acetamide-salicylic acid (ASL) and 5-benzamide-salicylic acid (BSL), present good capacity as free-radical scavengers in the AAPH-induced RBC hemolysis. The single electron transfer results indicate that BSL acts as a more potent scavenger (due to the small hemolysis percentage) than ASL, in contrast with SAC that does not exhibit scavenger capacity in this model in any evaluated concentrations. These results are in accordance to 2,2-diphenyl-1-picrylhydrazyl (DPPH) evaluation. ${ }^{14}$

As can be seen from Table 1, the HAT value of the benzoylic compound BZL showed an energy of $25.46 \mathrm{kcal} \mathrm{mol}^{-1}$. The derivatives obtained by molecular association, ASL and BSL, have lower energies when compared to SAC of 39.71 and $38.85 \mathrm{kcal} \mathrm{mol}^{-1}$, respectively.

The structure-capacity relationship of $p$-aminophenol and salicylate association derivatives using five phenolic derivatives: ACP, SAC, BZL, ASL, and BSL showed that these compounds, that here are assessed for their antioxidant capacity in human erythrocytes, differ mainly in the carbonyl or acylamide substituent group at the ortho and para positions, respectively.

Furthermore, the antioxidant or pro-oxidant effects of these molecules are also related to its $\mathrm{p} K_{\mathrm{a}}$ values. The $\mathrm{p} K_{\mathrm{a}}$ values for Trolox, acetaminophen, and salicylic acid are 12.1 (TLX), 9.51 (ACP), 3.0 (SAC), and $<6.0$ (phenol). ${ }^{27,28}$
Therefore, acetamide group in para position of phenol (6.0) increase $\mathrm{p} K_{\mathrm{a}}$ values (9.51 of ACP) and the low $\mathrm{p} K_{\mathrm{a}}$ values are related to pro-oxidant effect such as salicylic acid, while high $\mathrm{p} K_{\mathrm{a}}$ values are related to antioxidant effect such as Trolox and acetaminophen. So, the change of acetamide to benzylamide moiety on acetaminophen or the addition of acetamide or benzylamide function at the 5-position seems to increase the SAC protective capacity of these molecules by their higher expected $\mathrm{p} K_{\mathrm{a}}$ when compared to SAC.

Our antioxidant mechanism is a little bit different from other previously reported ${ }^{29-32}$ due to carboxyl moiety. In accordance with SET and HAT mechanisms, the SPLET mechanism showed the same performance. ACP and BZL have the low SPLET values compared to TLX $\left(38.43 \mathrm{kcal} \mathrm{mol}^{-1}\right)$ of 48.19 and $51.32 \mathrm{kcal} \mathrm{mol}^{-1}$, respectively, as can be seen from Table 1. In addition, the results for salicylic acid and its derivatives are not in agreement with biological effects. The SPLET values of our derivatives obtained by molecular association, ASL and BSL, have lower energies when compared to SAC $\left(76.54 \mathrm{kcal} \mathrm{mol}^{-1}\right)$ of 70.50 and $73.43 \mathrm{kcal} \mathrm{mol}^{-1}$, respectively. Therefore, we could have a complementary mechanism for SPLET.

A hypothetical radical pathway for the SPLET of salicylic acid and its amide derivatives was proposed (Figure 4). First, an initial proton loss (a) followed by an electron abstraction (b) to form semiquinone (c). The positive radical charge is stabilized by $[1,3]$ tautomerization (d). Additionally, we suggest on the semiquinone form a conventional [1,5] hydrogen shift between phenol and carbonyl groups (e) and the antioxidant capacity depends on their chemical stability. We can see that the positive radical charge is delocalized by resonance only at the carboxyl moiety before the $[1,5]$ hydrogen shift and after that, the number of resonance structure increase due to phenoxyl, benzene, and nitrogen moieties by means of $[1,3]$ and $[1,2]$ tautomerizations. This antioxidant mechanism was previously reported by Borges et al..$^{33}$

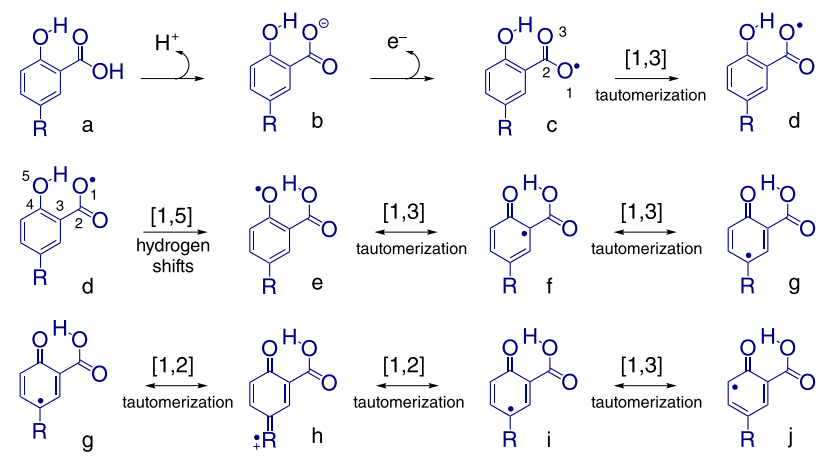

Figure 4. Hypothetical SPLET pathway for salicylic acid derivatives. 
In fact, the recombination reaction by [1,5] hydrogen shift between carbonyl and phenol groups is thermodynamically favored in $-18.05 \mathrm{kcal} \mathrm{mol}^{-1}$ for SAC, as described in Figure 5. The ASL and BSL have more favored energy barriers of -21.16 and $-24.40 \mathrm{kcal} \mathrm{mol}^{-1}$, respectively.
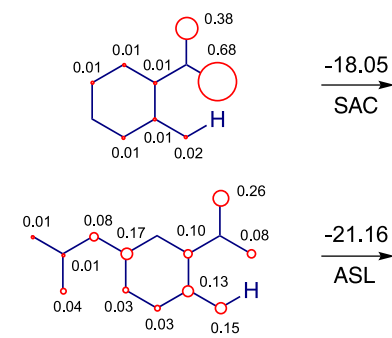

$$
\underset{\mathrm{ASL}}{\stackrel{-21.16}{\longrightarrow}}
$$

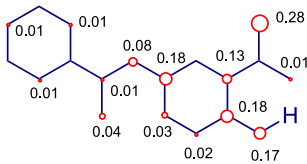

$$
\underset{\mathrm{BSL}}{\stackrel{-24.40}{\longrightarrow}}
$$

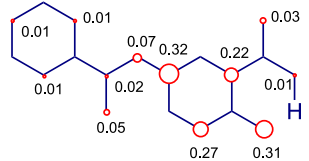

Figure 5. Energy barriers and spin density contributions for carbonyl and phenoxyl free radicals of the salicylates derivatives studied here.

The calculated spin density to sequential proton loss electron transfer (SPLET) for salicylic acid and its associated derivatives shows the main contributions from the carboxyl moiety and phenoxyl for all derivatives before and after the [1,5] hydrogen shifts, respectively (Figure 5). The amide and aromatic contributions decrease in ASL and BSL. After [1,5] hydrogen shifts, all contribution in phenoxyl and aromatic positions especially in carbon atoms at ortho and para positions are more expressive when compared to carbonyl group. In semiquinone forms, these positions are of 0.38 (oxygen), 0.28 and 0.30 (carbon atoms at ortho positions), and 0.41 (carbon atom at para position). These contributions decrease a little bit on ASL and BSL derivatives for 0.32 (oxygen), 0.23 and 0.24 (carbon atoms at ortho positions), and 0.33 (carbon atom at para position) and 0.31 (oxygen), 0.22 and 0.27 (carbon atoms at ortho positions), and 0.32 (carbon atom at para position), respectively.

These contributions show the following chemical stability order: $\mathrm{BSL}>\mathrm{ASL}>\mathrm{SAC}$, in accordance to the biological evaluation. Also, ASL and BSL are chemical analogous to GAC, a hydroxylated metabolic derivative of SAC. In fact, the GAC have been reported ${ }^{14,34}$ to be effective as antioxidant. Therefore, our new derivatives have more protective capacity when compared to salicylic acid and easy to be obtained and, therefore, they might serve as model molecules for the design of antioxidant candidates related to salicylates.

\section{Conclusions}

Phenolic compounds showed very different activities as free-radical scavengers and AAPH-induced RBC hemolysis. Benzaminophen showed a potential scavenger capacity or small hemolysis percentage when compared to acetaminophen. The $p$-aminophenol and salicylate association derivatives have more protective effect as free-radical scavengers than salicylic acid. Acetamide or benzamide at para position decreased the single electron transfer and hydrogen atom transfer values resulting in a better scavenger capacity. The cytoprotective effect can be related to sequential proton loss electron transfer (SPLET) increasing their antioxidant capacity. The SPLET mechanism depends on their [1,5] hydrogen shifts between carboxyl and phenol moieties and chemical stability before and after that. The molecular association approach provides protective compounds more effective than SAC as antioxidant in human erythrocytes.

\section{Acknowledgments}

The authors would like to thank the CNPq (Brazilian Agency) for the financial support.

\section{Author Contributions}

Valéria A. Barros was responsible for the data curation; Glaécia A. N. Pereira for the investigation; Sirlene S. B. Ota for the formal analysis; Fernanda P. A. Melo for the validation; Ana Carolina S. P. S. de Jesus for the visualization; Anderson B. Lima for the resources; Albérico B. F. da Silva for the writing review and editing; Rosivaldo S. Borges for the conceptualization and writing original draft.

\section{References}

1. Kawasaki, T.; Ozeki, Y.; Igawa, T.; Kambayashi, J.; Stroke 2000, 31, 591.

2. Gum, P. A.; Kottke-Marchant, K.; Poggio, E. D.; Gurm, H.; Welsh, P. A.; Brooks, L.; Sapp, S. K.; Topol, E. J.; Am. J. Cardiol. 2001, 88, 230.

3. Morrow, J. D.; Hill, K. E.; Burk, R. F.; Nammour, T. M.; Badr, K. F.; Roberts, L. J.; Proc. Natl. Acad. Sci. U. S. A. 1990, 87, 9383.

4. Davi, G.; Gresele, P.; Violi, F.; Basii, S.; Catalano, M.; Giammarresi, C.; Volpato, R.; Nenci, G. G.; Ciabattoni, G.; Patrono, C.; Circulation 1997, 96, 69.

5. Patrono, C.; Fitz Gerald, G. A.; Arterioscler., Thromb., Vasc. Biol. 1997, 17, 2309.

6. Voutilainen, S.; Morrow, J. D.; Roberts, L. J.; Alfthan, G.; Alho, H.; Nyyssonen, K.; Salonen, J. T.; Arterioscler., Thromb., Vasc. Biol. 1999, 19, 1263. 
7. Kettle, A. J.; Gedye, C. A.; Winterbourn, C. C.; Biochem. Pharmacol. 1993, 45, 2003.

8. Dinis, T. C. P.; Madeira, V. M. C.; Almeida, L. M.; Arch. Biochem. Biophys. 1994, 315, 161.

9. Ramos, C. L.; Pou, S.; Rosen, G. M.; Biochem. Pharmacol. 1995, 49, 1079.

10. Maharaj, D. S.; Saravanan, K. S.; Maharaj, H.; Mohanakumar, K. P.; Daya, S.; Neurochem. Int. 2004, 44, 355.

11. Wallace, J. L.; Physiol. Rev. 2008, 88, 1547.

12. Ivanov, I. T.; Biochim. Biophys. Acta, Biomembr. 1999, 1415, 349.

13. Kuang, Z. H.; Wang, P. F.; Zheng, R. L.; Liu, Z. L.; Liu, Y. C.; Chem. Phys. Lipids 1994, 71, 95.

14. Borges, R. S.; Pereira, G. A. N.; Vale, J. K. L.; França, L. C. S.; Monteiro, M. C.; Alves, C. N.; da Silva, A. B. F.; Chem. Biol. Drug Des. 2013, 81, 414.

15. Liu, Z. Q.; Han, K.; Lin, Y. J.; Luo, X. Y.; Biochim. Biophys. Acta 2002, 1570, 97.

16. Jia, Z. S.; Zhou, B.; Yang, L.; Wu, L. M.; Liu, Z. L.; Chin. J. Magn. Reson. 1998, 15, 23.

17. Liu, Z. Q.; Ma, L. P.; Zhou, B.; Yang, L.; Liu, Z. L.; Chem. Phys. Lipids 2000, 106, 53.

18. Frisch, M. J.; Trucks, G. W.; Schlegel, H. B.; Scuseria, G. E.; Robb, M. A.; Cheeseman, J. R.; Scalmani, G.; Barone, V.; Mennucci, B.; Petersson, G. A.; Nakatsuji, H.; Caricato, M.; Li, X.; Hratchian, H. P.; Izmaylov, A. F.; Bloino, J.; Zheng, G.; Sonnenberg, J. L.; Hada, M.; Ehara, M.; Toyota, K.; Fukuda, R.; Hasegawa, J.; Ishida, M.; Nakajima, T.; Honda, Y.; Kitao, O.; Nakai, H.; Vreven, T.; Montgomery Jr., J. A.; Peralta, J.; Ogliaro, F.; Bearpark, M.; Heyd, J. J.; Brothers, E.; Kudin, K. N.; Staroverov, V. N.; Keith, T.; Kobayashi, R.; Normand, J.; Raghavachari, K.; Rendell, A.; Burant, J. C.; Iyengar, S. S.; Tomasi, J.; Cossi, M.; Rega, N.; Millam, J. M.; Klene, M.; Knox, J. E.; Cross, J. B.; Bakken, V.; Adamo, C.; Jaramillo, J.; Gomperts, R.; Stratmann, R. E.; Yazyev, O.; Austin, A. J.; Cammi, R.; Pomelli, C.; Ochterski, J. W.; Martin, R. L.; Morokuma, K.; Zakrzewski, V. G.; Voth, G. A.; Salvador, P.;
Dannenberg, J. J.; Dapprich, S.; Daniels, A. D.; Farkas, O.; Foresman, J. B.; Ortiz, J. V.; Cioslowski, J.; Fox, D. J.; Gaussian 09, Revision C.02; Gaussian, Inc., Wallingford, CT, USA, 2009

19. Parr, R. G.; Yang, W.; Density Functional Theory of Atoms and Molecules; Oxford University Press: New York, 1989.

20. Stewart, J. J. P.; J. Comput. Chem. 1989, 10, 209.

21. Becke, A. D.; Phys. Rev. A 1988, 38, 3098.

22. Lee, C.; Yang, W.; Parr, R. G.; Phys. Rev. B 1988, 37, 785.

23. Sevanian, A.; Ursini, F.; Free Rad. Biol. Med. 2000, 29, 306.

24. Takebayashi, J.; Kaji, H.; Ichiyama, K.; Makino, K.; Gohda, E.; Yamamoto, I.; Tai, A.; Free Rad. Biol. Med. 2007, 43, 1156.

25. Laranjinha, J.; Vieira, O.; Madeira, V.; Almeida, L.; Arch. Biochem. Biophys. 1995, 323, 373.

26. Mendiratta, S.; Qu, Z. C.; May, J. M.; Free Rad. Biol. Med. 1998, 24, 789.

27. Williams, D. A.; Lemke, T. L.; Foye's Principles of Medicinal Chemistry, $6^{\text {th }}$ ed.; Lippincott Williams and Wilkins: Philadelphia, 2007.

28. Warren, J. J.; Mayer, J. M.; Proc. Natl. Acad. Sci. U. S. A. 2010, 107,5282 .

29. Galano, A.; Mazzone, G.; Alvarez-Diduk, R.; Marino, T.; Alvarez-Idaboy, J. R.; Russo, N.; Annu. Rev. Food Sci. Technol. 2016, 7, 335 .

30. Alberto, M. E.; Russo, N.; Grand, A.; Galano, A.; Phys. Chem. Chem. Phys. 2013, 15, 4642.

31. Leopoldini, M.; Russo, N.; Toscano, M.; Food Chem. 2011, 125, 288

32. Marino, T.; Galano, A.; Russo, N.; J. Phys. Chem. B 2014, 118 , 10380.

33. Borges, R. S.; Mendes, A. P. S.; Silva, B. H. S.; Alves, C. N.; do Nascimento, J. L. M.; Med. Chem. Res. 2011, 20, 269.

34. Borges, R. S.; Castle, S. L.; Bioorg. Med. Chem. Lett. 2015, 25, 4808.

Submitted: October 16, 2020

Published online: March 8, 2021 